\title{
Using ultrasound radio frequency technology to assess regression of the structure and function of the carotid artery by radioiodine therapy in hyperthyroidism patients
}

Chunsong Kang

Shanxi Academy of Medical Sciences and Shanxi DAYI Hospital, Taiyuan, China

Submitted: 1 April 2013

Accepted: 16 August 2013

Arch Med Sci 2015; 11, 6: 1236-1243

DOI: 10.5114 /aoms.2015.53294

Copyright $\odot 2015$ Termedia \& Banach

\section{Abstract}

Introduction: The aim of the study was to investigate the structure and function of the carotid artery in patients with hyperthyroidism by ultrasound radio frequency data technology (RF data) and the effect of ${ }^{131}$ on them.

Material and methods: Seventy patients with primary hyperthyroidism and 74 healthy volunteers were enrolled in this study. Structural and functional parameters of the common carotid artery were measured in every patient before and after ${ }^{131}$ I treatment through the RF data, such as intima media thickness (IMT), functional compliance coefficient (CC), stiffness index ( $\beta$ ), and pulse wave velocity (PWV). We also analyzed the correlation between these parameters and patients' age, body mass index, hemodynamic parameters (blood pressure, heart rate), thyroid hormone levels and other risk factors. Results: There was a significant difference in IMT between hyperthyroid patients and the control group at baseline (483.6 vs. $443.3 \mu \mathrm{m}, p<0.01)$; after treatment, the IMT decreased significantly (428.7 vs. $483.6 \mu \mathrm{m}, p<0.001$ ). Furthermore, the IMT was correlated with patients' age and systolic blood pressure $(r=0.525, p<0.01$ and $r=0.289, p<0.05$, respectively). The $\beta$ and PWV were also higher than the control group $(7.26 \mathrm{vs} .5 .87,6.27 \mathrm{vs} .5 .57 \mathrm{~m} / \mathrm{s}$, respectively; all $p<0.001$ ); CC was lower than the control group (0.98 vs. $\left.1.19 \mathrm{~mm}^{2} / \mathrm{KPa}, p<0.01\right)$; after treatment, $\mathrm{PWV}$ and $\beta$ were lower than baseline (5.66 vs. $6.27,5.81$ vs. $7.26 \mathrm{~m} / \mathrm{s}$, respectively; all $p<0.01)$, and CC was higher than baseline. In addition, they were significantly correlated with age $(r=0.525, p<0.01$ and $r=0.289, p<0.05$, respectively). However, these parameters were not correlated with the level of thyroid hormones.

Conclusions: Six-month ${ }^{131}$ I treatment for patients with hyperthyroidism reverses the structural and functional damage in the carotid artery, which is sensitively evaluated by the RF data technique.

Key words: ultrasonic examination, hyperthyroidism, carotid artery.

\section{Introduction}

Hyperthyroidism is closely related with cardiovascular disease [1]. Excessive secretion of thyroid hormones results in a highly dynamic state, and further leads to cardiovascular disorders [2]. The structure and function of arteries are closely related to hypertension, atherosclerosis, and ultimately to cardiovascular and cerebrovascular events. The carotid artery is an elastic blood vessel, and whether its structure and function are damaged in patients with hyperthyroidism and its ability to recover after treatment are critically important to control related cardiovascular

\author{
Corresponding author: \\ Chunsong Kang \\ Shanxi Academy \\ of Medical Sciences \\ and Shanxi DAYI Hospital \\ No. 99, Longcheng Da Jie \\ 030032 Taiyuan, China \\ Phone: +8613834518776 \\ E-mail: jiwen0004@126.com
}


events. Up to now, most research has focused on cardiac function in patients with hyperthyroidism; however, few studies are related to vascular function. In addition, morphological and functional changes of the carotid artery are not completely parallel in clinical practice. Therefore, a combination of both should be investigated to fully understand vascular lesions [3].

Radioactive iodine 131 therapy is a treatment for hyperthyroidism. When a small dose of ${ }^{131}$ is swallowed, it is absorbed into the bloodstream in the gastrointestinal tract and concentrated from the blood by the thyroid gland, where it begins destroying the gland's cells [4]. Other medical treatments for hyperthyroidism might be focused on the apoptotic pathway [5].

Compliance coefficient (CC), stiffness index ( $\beta$ ) and other elastic parameters are objective ways to demonstrate arteriosclerosis, whose changes are of great significance to the early evaluation of arterial elasticity. Pulse wave velocity (PWV) is also believed by most researchers to be a reliable arterial elasticity parameter [6, 7]. Furthermore, the intima media thickness (IMT) of the carotid artery is proved to be an independent predictor of cardiovascular and cerebrovascular events [8]. In this study, we applied radio frequency (RF) data technology to non-invasively observe a structural parameter (IMT) and functional parameters (CC, $\beta$, PWV) in patients with hyperthyroidism to evaluate whether structural and functional damage occurred in patients with hyperthyroidism compared with healthy controls and, if damaged, whether treatment with ${ }^{131}$ I would recover the damage.

\section{Material and methods}

\section{Subjects}

The study population was selected from a University Hospital endocrinological outpatient clinic between 2011 and 2012. Patients with coexisting hypertension, diabetes mellitus, coronary artery disease, obesity or hyperlipidemia and patients who had received any previous treatment for thyroid disorder were excluded. Informed consent was obtained from all patients on inclusion in the study. Clinical, laboratory and ultrasound data of the patients were compared with 74 healthy controls from the same population. Patients were followed up 6 months prospectively and clinical, laboratory and ultrasonography data were recorded.

\section{Clinical assessment}

Serum thyroid hormones were detected by radioimmunoassay (normal values: FT3 > $6.8 \mathrm{pmol} / \mathrm{l}$, FT4 $>23.0 \mathrm{pmol} / \mathrm{l}, \mathrm{TSH}<0.27 \mu \mathrm{lU} / \mathrm{ml}, 24 \mathrm{~h}{ }^{131} \mathrm{l}$ absorption rate $>40 \%$ ). Hyperthyroidism was diagnosed through laboratory analysis, including thyroid ultra- sound, and clinical signs. Many drugs could influence the vascular system (statins, antihypertensive drugs, etc), but none of the patients in the study used any medication other than ${ }^{131}$ i; thus, we excluded the possible effects of some medicines such as statins, antihypertensive drugs or amiodarone.

\section{General information}

The subjects' height, body mass, body mass index, blood pressure and heart rate were collected in the outpatient clinic. The blood tests for serum thyroid hormone levels and 24-hour iodine uptake rate were collected as well.

\section{Measurement of parameters of carotid artery}

We used the Mylab70 color Doppler ultrasound device (Esaote, Maastricht, Netherlands) with a 75 high-sensitive 4-13 MHz multi-frequency linear array probe transducer. The patient is in the supine position with his or her head $20^{\circ}$ deviated to the opposite side. The device has built-in digital system processing software. A series of carotid artery parameters such as RF quality intima-media thickness (RFQIMT), RF quality arterial stiffness (RFQAS), real-time carotid IMT and elasticity can be measured automatically. Structural and functional parameters of the carotid artery were acquired as follows: the subjects were set in a supine position, fully exposing their neck. The bilateral long axes of the carotid arteries were explored, then the probe was adjusted so that the ultrasound beam was perpendicular to the anterior and posterior wall of the carotid artery and to get a clear image of the front and rear walls of the blood vessels. Then RFQIMT was initiated, the carotid artery IMT was measured at $1.0-1.5 \mathrm{~cm}$ at the posterior wall of the carotid artery enlargement proximal end, quantitative real-time detection of six cardiac cycles of carotid artery IMT was performed, then their mean values were calculated. The system can automatically calculate the PWV, hardness parameter $(\beta)$ and arterial compliance (CC). Parameter definitions: $\beta=\ln$ $(P s / P d) /[(D s-D d) / D d] ; C C=\pi(D s \times D s-D d \times D d) /$ $[4(P s-P d)] ; P W V=\sqrt{ }(\beta P d / 2 \rho)$. Among them, $D s$ is the largest internal diameter of the carotid artery, $D d$ is the minimum inside diameter of the carotid artery, and $\rho$ is the density of blood.

\section{Statistical analysis}

Baseline and descriptive data are presented as mean \pm standard deviation, Among the three groups, analysis of variance (ANOVA) was used and between-group analysis was performed using LSD $t$-test. The left and right side comparison was analyzed using the paired $t$-test. Then, Pearson correlation analysis was used to explore the cor- 
relation between these parameters. The repeatability was evaluated with a Bland-Altman analysis diagram. Value of $p<0.05$ was considered statistically significant and SPSS 13.0 was used for statistical calculations (SPSS, Chicago, IL).

\section{Results}

The baseline clinical and laboratory characteristics of the three groups are presented in Table I. Age and gender distribution were similar among the three groups $(p>0.05)$. Seventy patients with hyperthyroidism completed the study. Euthyroidism was achieved in 32 patients. Other 38 patients remained hyperthyroid and were evaluated for other therapies, including propylthiouracil or surgery. The 32 patients who achieved euthyroidism were followed up for 6 months after ${ }^{131}$ I was discontinued, and all remained euthyroid during this period. Before treatment, patients' systolic blood pressure, heart rate, FT3 and FT4 were higher than the control group $(p<0.01)$, whereas the TSH was lower compared to the control group ( $p<0.01$ ). After treatment, FT3, FT4, heart rate and systolic pressure was lower than before treatment, while TSH was higher than before treatment $(p<0.01)$. Furthermore, after treatment, patients' diastolic blood pressure, systolic blood pressure, pulse pressure, heart rate, FT3, FT4 and TSH were similar to those in the control group, the difference was not statistically significant $(p>0.05)$ (Table I).

\section{Comparison and correlation analysis of structural parameter in right carotid artery in three groups}

There were no differences in IMT, CC, $\beta$ and PWV between the left and right common carotid artery between the three groups, as presented in Table II.
Carotid IMT was significantly higher in patients with hyperthyroidism than in healthy controls $(p<0.05)$. After 6 months of treatment, carotid IMT decreased significantly $(p<0.01)$, and there was no difference in the carotid IMT between the control and after treatment (Table III, Figure 1). Further analysis showed that carotid IMT was positively correlated with age and systolic blood pressure $(r=0.525, p<0.01, r=0.289, p<0.05)$ (Table IV). After controlling for age, IMT was not correlated with body mass index, blood pressure, pulse pressure, heart rate or thyroid hormone levels $(p>0.05)$ (Table V).

\section{Comparison and correlation analysis of functional parameters in right carotid artery in three groups}

Before treatment, CC of the carotid artery was lower than the control group, while $\beta$ and PWV were higher than those in the control group $(p<0.01)$. After 6 months of treatment, CC, $\beta$ and PWV had recovered to the normal level, and there were no differences between the post-treatment group and the healthy controls $(p>0.05)$ (Table III, Figure 1).

In addition, CC, $\beta$ and PWV were significantly related to patients' age $(r=-0.345 ; r=0.502$; $r=0.540, p<0.01$ ). Body mass index, systolic blood pressure, diastolic blood pressure, pulse pressure, heart rate and thyroid hormone levels had no correlation $(p>0.05)$ (Table IV). After controlling for age, CC and diastolic blood pressure were positively correlated $(r=0.392, p<0.01)$ and heart rate was negatively correlated $(r=-0.294$, $p<0.05) ; \beta$ and diastolic blood pressure were negatively correlated $(r=-0.440, p<0.01)$; PWV and pulse pressure, and heart rate were positively correlated $(r=0.303 ; r=0.295, p<0.05)$ (Table V).

Table I. Characteristics of three groups

\begin{tabular}{|c|c|c|c|c|c|}
\hline Parameter & $\begin{array}{l}\text { Controls } \\
\text { (74 cases) }\end{array}$ & $\begin{array}{l}\text { Before treatment } \\
\quad \text { (70 cases) }\end{array}$ & $\begin{array}{l}\text { After treatment } \\
\text { (32 cases) }\end{array}$ & $F\left(\chi^{2}\right)$ & Value of $p$ \\
\hline Age [years] & $34.1 \pm 10.0$ & $37.3 \pm 12.1$ & $33.39 \pm 11.1$ & 1.902 & 0.152 \\
\hline Gender (male/female) & $22 / 52$ & $22 / 48$ & $8 / 24$ & 0.436 & 0.804 \\
\hline BMI $\left[\mathrm{kg} / \mathrm{m}^{2}\right]$ & $22.48 \pm 2.41$ & $20.45 \pm 3.60^{\wedge}$ & $22.80 \pm 3.11^{*}$ & 10.365 & $<0.001$ \\
\hline $\mathrm{SBP}[\mathrm{mm} \mathrm{Hg}]$ & $113.96 \pm 10.64$ & $121.42 \pm 12.76^{\mathbf{\Delta}}$ & $117.45 \pm 8.48$ & 7.946 & 0.001 \\
\hline $\mathrm{DBP}[\mathrm{mm} \mathrm{Hg}]$ & $74.77 \pm 8.46$ & $70.94 \pm 9.36^{\wedge}$ & $77.45 \pm 6.83^{*}$ & 7.542 & $<0.001$ \\
\hline $\mathrm{PP}[\mathrm{mm} \mathrm{Hg}]$ & $39.19 \pm 6.80$ & $49.76 \pm 11.02$ & $40.97 \pm 8.38^{\star}$ & 26.828 & $<0.001$ \\
\hline $\mathrm{HR}[\mathrm{bpm}]$ & $68.61 \pm 10.28$ & $95.77 \pm 15.52^{\Delta}$ & $73.73 \pm 11.78^{*}$ & 85.035 & $<0.001$ \\
\hline FT3 [pmol/l] & $5.29 \pm 1.62$ & $30.57 \pm 16.75^{\wedge}$ & $6.18 \pm 4.57^{\star}$ & 102.626 & $<0.001$ \\
\hline FT4 [pmol/ll] & $16.34 \pm 4.34$ & $66.55 \pm 33.42^{\Delta}$ & $20.10 \pm 15.93^{*}$ & 102.866 & $<0.001$ \\
\hline $\mathrm{TSH}[\mu \mathrm{IU} / \mathrm{ml}]$ & $1.77 \pm 1.05$ & $0.07 \pm 0.10^{\star}$ & $2.35 \pm 7.40^{*}$ & 14.526 & $<0.001$ \\
\hline
\end{tabular}

${ }^{*} p<0.01$, comparison between before treatment and after treatment; ${ }^{\mathbf{A}} p<0.01$, comparison between controls and pre-treatment group. 
Table II. Structural and functional parameters of bilateral carotid arteries in three groups $(\bar{x} \pm s)$

\begin{tabular}{|c|c|c|c|c|}
\hline Parameter & Right side & Left side & Value of $t$ & Value of $p$ \\
\hline \multicolumn{5}{|l|}{ Controls: } \\
\hline $\mathrm{IMT}[\mu \mathrm{m}]$ & $443.30 \pm 81.55$ & $449.33 \pm 106.48$ & -0.559 & 0.578 \\
\hline $\mathrm{CC}\left[\mathrm{mm}^{2} / \mathrm{KPa}\right]$ & $1.19 \pm 0.38$ & $1.17 \pm 0.42$ & 0.668 & 0.507 \\
\hline$\beta$ & $5.81 \pm 1.94$ & $6.13 \pm 2.42$ & -1.230 & 0.223 \\
\hline $\mathrm{PWV}[\mathrm{m} / \mathrm{s}]$ & $5.57 \pm 0.96$ & $5.65 \pm 1.19$ & -0.802 & 0.425 \\
\hline \multicolumn{5}{|l|}{ Before treatment: } \\
\hline $\mathrm{IMT}[\mu \mathrm{m}]$ & $483.57 \pm 97.31$ & $479.21 \pm 83.59$ & 0.300 & 0.765 \\
\hline $\mathrm{CC}\left[\mathrm{mm}^{2} / \mathrm{KP}\right]$ & $0.98 \pm 0.37$ & $1.03 \pm 0.35$ & -0.950 & 0.345 \\
\hline$\beta$ & $7.26 \pm 2.48$ & $7.16 \pm 2.79$ & 0.111 & 0.912 \\
\hline $\mathrm{PWV}[\mathrm{m} / \mathrm{s}]$ & $6.27 \pm 1.09$ & $6.16 \pm 1.19$ & 0.623 & 0.535 \\
\hline \multicolumn{5}{|l|}{ After treatment: } \\
\hline $\mathrm{IMT}[\mu \mathrm{m}]$ & $428.66 \pm 98.32$ & $451.34 \pm 101.23$ & -1.740 & 0.092 \\
\hline $\mathrm{CC}\left[\mathrm{mm}^{2} / \mathrm{KPa}\right]$ & $1.29 \pm 0.44$ & $1.26 \pm 0.38$ & 0.658 & 0.515 \\
\hline$\beta$ & $5.81 \pm 2.23$ & $5.66 \pm 1.99$ & 0.450 & 0.656 \\
\hline $\mathrm{PWV}[\mathrm{m} / \mathrm{s}]$ & $5.66 \pm 1.10$ & $5.60 \pm 0.99$ & 0.409 & 0.686 \\
\hline
\end{tabular}

IMT - Intima media thickness, CC - compliance coefficient, $\beta$ - stiffness index, PWV - pulse wave velocity.

Table III. Comparisons of structural and functional parameters of carotid artery among three groups $(\bar{x} \pm s)$

\begin{tabular}{|lccccc|}
\hline Parameter & $\begin{array}{c}\text { Controls } \\
(74 \text { cases })\end{array}$ & $\begin{array}{c}\text { Before treatment } \\
(70 \text { cases })\end{array}$ & $\begin{array}{c}\text { After treatment } \\
(32 \text { cases })\end{array}$ & Value of $F$ & Value of $p$ \\
\hline IMT $[\mu \mathrm{m}]$ & $443.30 \pm 81.55$ & $483.57 \pm 97.31^{\star}$ & $428.66 \pm 98.32^{*}$ & 5.214 & $<.006$ \\
\hline CC $\left[\mathrm{mm}^{2} / \mathrm{KPa}\right]$ & $1.19 \pm 0.38$ & $0.98 \pm 0.37^{*}$ & $1.29 \pm 0.44^{*}$ & 8.772 & 8.287 \\
\hline$\beta$ & $5.87 \pm 1.94$ & $7.26 \pm 2.48^{*}$ & $5.81 \pm 2.23^{*}$ & $8.502<001$ \\
\hline PWV $[\mathrm{m} / \mathrm{s}]$ & $5.57 \pm 0.98$ & $6.27 \pm 1.09^{*}$ & $5.66 \pm 1.10^{*}$ & $<001$ \\
\hline
\end{tabular}

${ }^{*} p<0.01$, comparison between before treatment and after treatment; ${ }^{\mathbf{A}} p<0.05$ comparison between controls and before treatment group.

\section{Repetitive inspection}

Two physicians measured the IMT and PWV correlation coefficient on the same subject: $r 1=0.97$, $r 2=0.93, p<0.001$. The mean difference was $-16.73 \pm 24.11 \mu \mathrm{m}$ and $0.11 \pm 0.28 \mathrm{~m} / \mathrm{s}$, respectively. Then, the same physician measured the IMT and PWV at different time points, showing the correlation coefficient: $r 1=0.97, r 2=0.94$, $p<0.001$, mean difference was $-12.33 \pm 35.33 \mu \mathrm{m}$ and $-0.08 \pm 0.19 \mathrm{~m} / \mathrm{s}$, respectively. Bland-Altman analysis illustrated a consistent trend in repeated measurements of structural and functional parameters of carotid arteries (Figures 2, 3).

\section{Discussion}

In this study, we found that patients with hyperthyroidism had significantly increased damage both in structure and function of the carotid ar- tery, and that treatment with ${ }^{131}$ I significantly reversed this damage in hyperthyroidism. Both the structural (IMT) and the functional parameters (CC, $\beta$ and PWV) were measured by the RF data technique in the present study.

Hemodynamic changes in thyroid hormone act on the circulatory system, leading to decreased peripheral resistance, increased heart rate, myocardial contractility and systolic blood pressure, thereby causing increased pulse pressure and greatly increased cardiac output $[9,10]$. Volzke et al. [11] found increased carotid IMT in the low TSH population, and there was a significant relation between advanced age, male sex, diabetes mellitus, hypertension, medication of hypertension and previous cardiovascular events. We also detected a positive relation with age, and because of our exclusion criteria, we did not investigate the relationship between the other chronic diseases. 
A

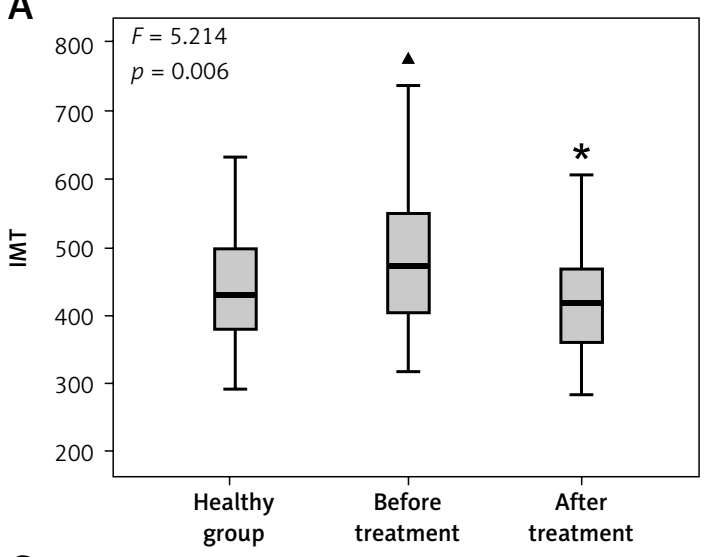

C

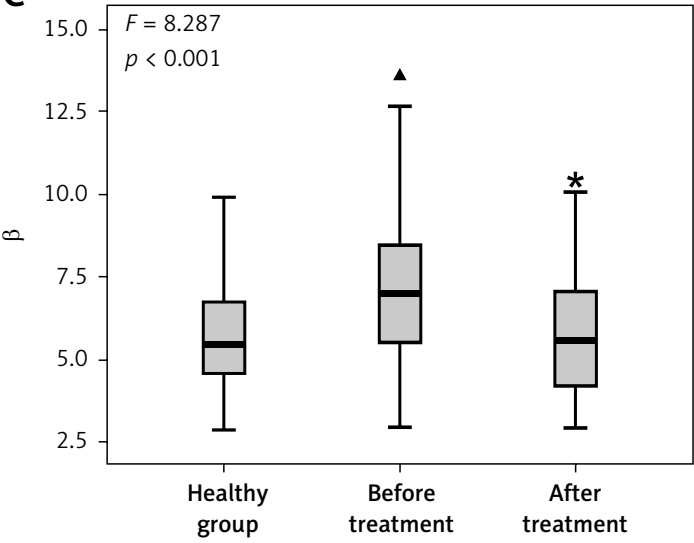

B

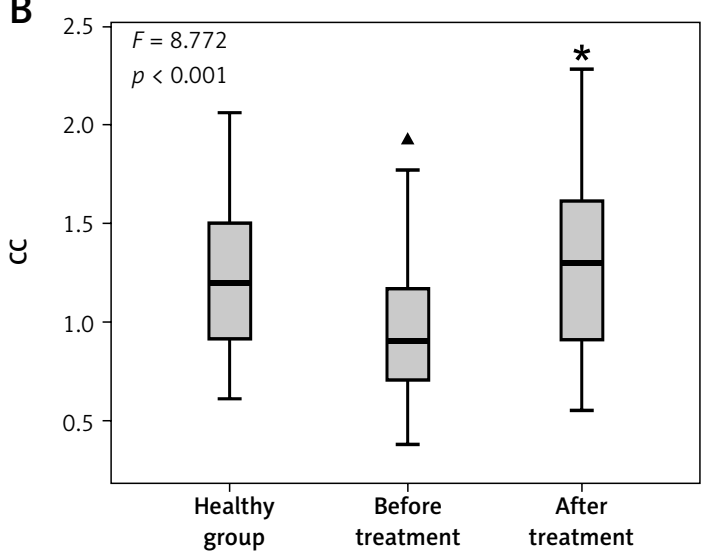

D

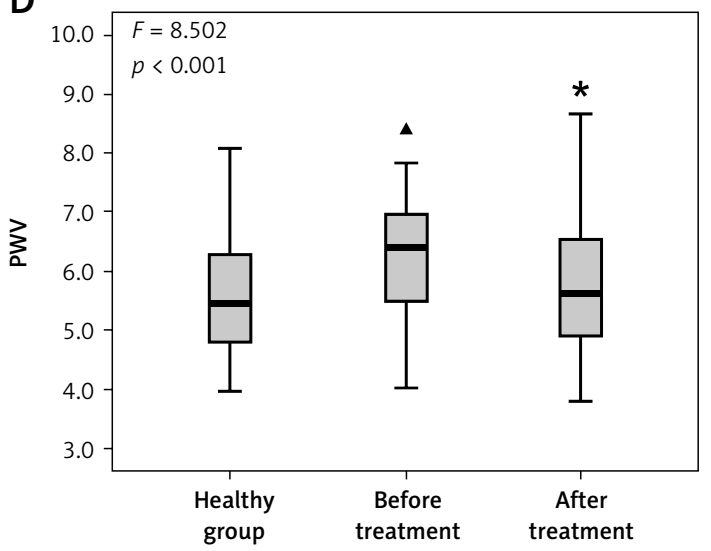

Figure 1. Comparisons of structural and functional parameters in three groups; the left bar is the control group, the middle bar is the pre-treatment group and the right bar is the post- treatment group

${ }^{*} p<0.01$, comparison between before treatment and after treatment; $\mathbf{\Delta}<<0.05$, comparison between controls and before treatment group.

Table IV. Correlational analysis of related parameters ( $r$ value)

\begin{tabular}{|c|c|c|c|c|}
\hline Parameter & IMT & $\mathrm{CC}$ & $\beta$ & PWV \\
\hline Age & $0.525^{* *}$ & $-0.345^{* \star}$ & $0.502^{* *}$ & $0.540^{* *}$ \\
\hline BMI & 0.079 & -0.049 & 0.152 & 0.216 \\
\hline SBP & $0.289^{*}$ & 0.063 & -0.057 & 0.168 \\
\hline DBP & 0.218 & 0.220 & -0.187 & 0.043 \\
\hline PP & 0.168 & -0.141 & 0.114 & 0.179 \\
\hline$H R$ & -0.124 & -0.089 & -0.011 & 0.005 \\
\hline FT3 & -0.105 & -0.030 & 0.031 & 0.045 \\
\hline FT4 & -0.237 & -0.048 & 0.033 & 0.053 \\
\hline TSH & -0.140 & -0.158 & 0.059 & 0.113 \\
\hline
\end{tabular}

${ }^{* *} p<0.01,{ }^{*} p<0.05$

As for the vascular change in hyperthyroidism, Bilir [12] and other researchers suggested that hyperthyroidism leads to obviously increased carotid IMT compared with healthy subjects. In the current study, we observed increased IMT in patients with hyperthyroidism, indicating the thickened intima-tunica media of the artery. The related mechanisms were: 1 ) tensional change of the intima-tunica media of the artery to decreased peripheral resistance, in order to maintain stability, and then the IMT increases [13]; 2) with the increase of the pulse pressure, the vessel walls suffer more shearing force from the shearing force, which causes injury of blood vessel walls, and further thick vascular membranes. Therefore, controlling blood pressure in patients with hyper- 
Table V. Correlational analysis of related parameters after controlling for age ( $r$ value)

\begin{tabular}{|lcccc|}
\hline Parameter & IMT & CC & $\boldsymbol{\beta}$ & PWV \\
\hline BMI & 0.019 & 0.037 & 0.061 & 0.076 \\
\hline SBP & 0.144 & 0.152 & -0.170 & 0.099 \\
\hline DBP & 0.091 & $0.392^{* *}$ & $-0.440^{* *}$ & -0.188 \\
\hline PP & 0.101 & -0.168 & 0.190 & $0.303^{*}$ \\
\hline HR & -0.108 & $-0.294^{*}$ & 0.231 & $0.295^{*}$ \\
\hline FT3 & -0.197 & -0.067 & 0.111 & 0.187 \\
\hline FT4 & -0.301 & -0.057 & 0.042 & 0.084 \\
\hline TSH & -0.159 & -0.145 & 0.035 & 0.118 \\
\hline
\end{tabular}

${ }^{* *} p<0.01,{ }^{*} p<0.05$.

A

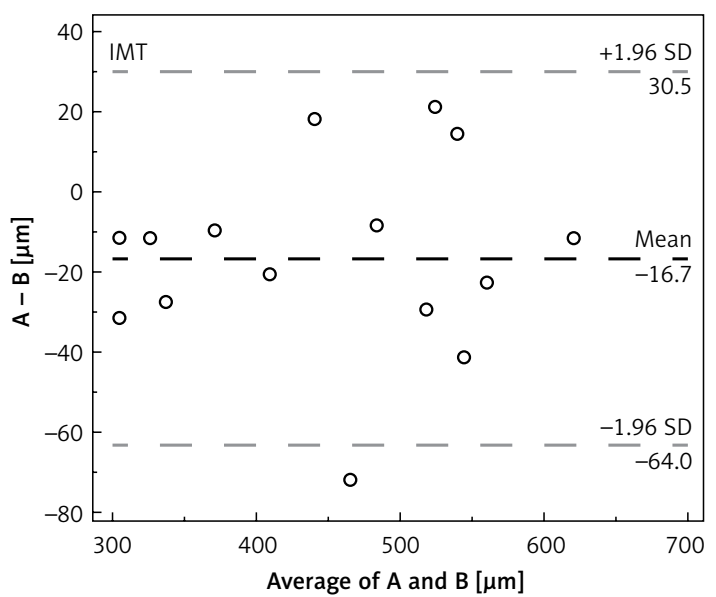

C

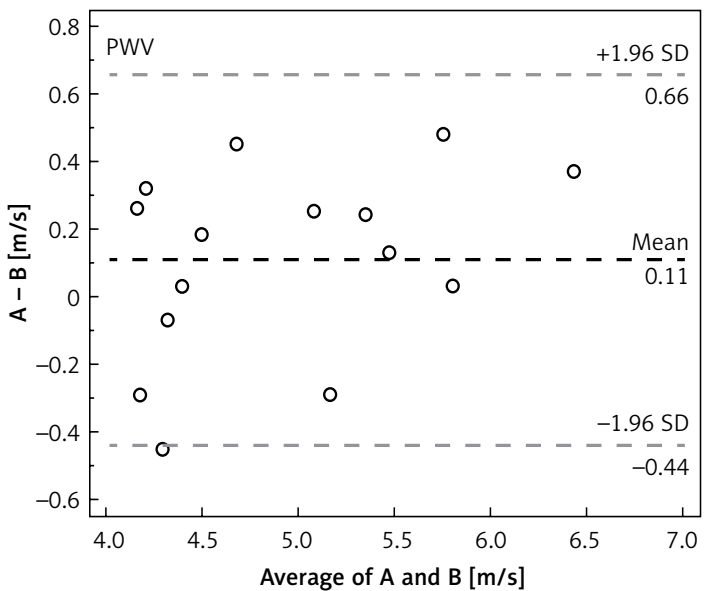

B

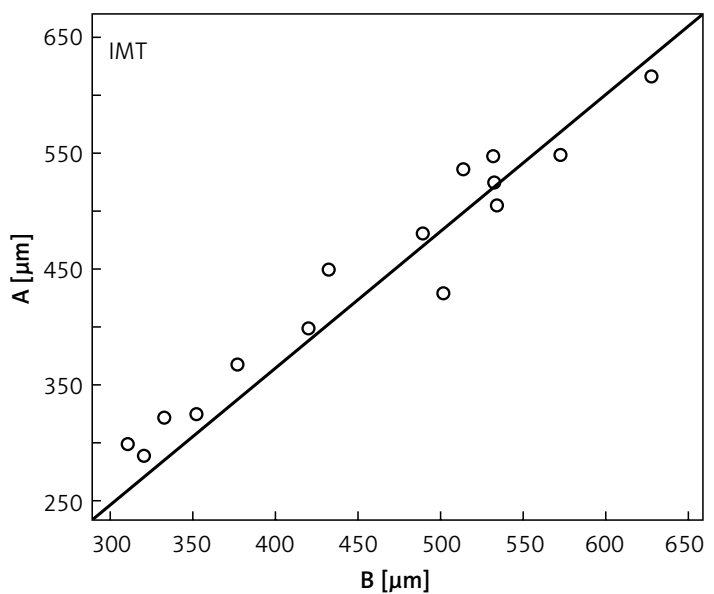

D

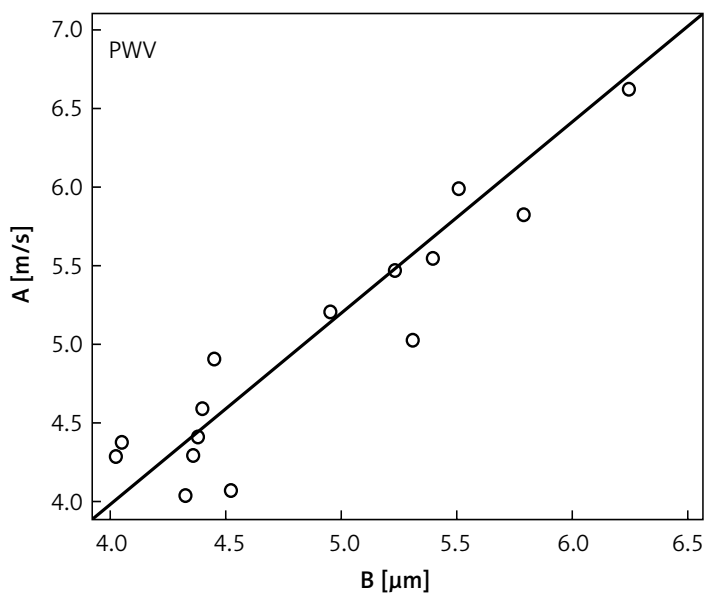

Figure 2. Two physicians measured the IMT and PWV correlation coefficient on the same subject $(\mathrm{A}, \mathrm{C}): r 1=0.97$ (B), $r 2=0.94(D)$

thyroidism is clinically significant, and to reduce cardiovascular and cerebrovascular complications.

Regarding the carotid function in hyperthyroidism, Inaba et al. [14] came to the conclusion that increased carotid artery stiffness in hyperthyroidism would recover with the decreased level of thyroid hormones. In our study, we found reduced CC and increased $\beta$ and PWV in patients with hyperthyroidism, suggesting reduced compliance and increased hardness. Possible mechanisms include: 1) increased cardiac output causes periodic expansion of vascular smooth muscle cells, resulting in decreased wall elasticity [15], increased vascular stiffness, and reduced compliance; 2 ) excessive 
A

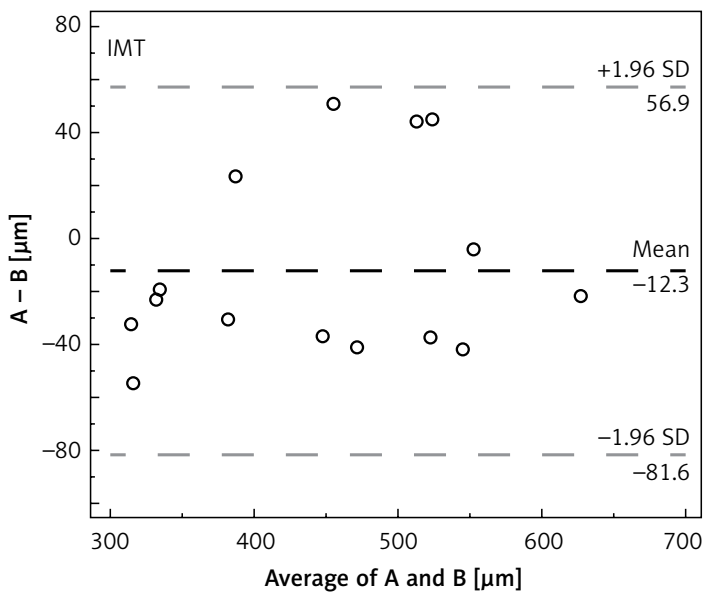

C

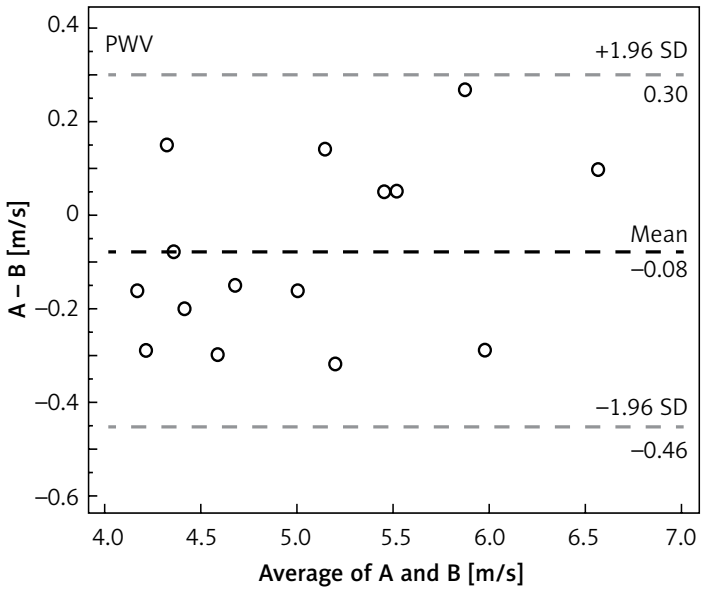

B

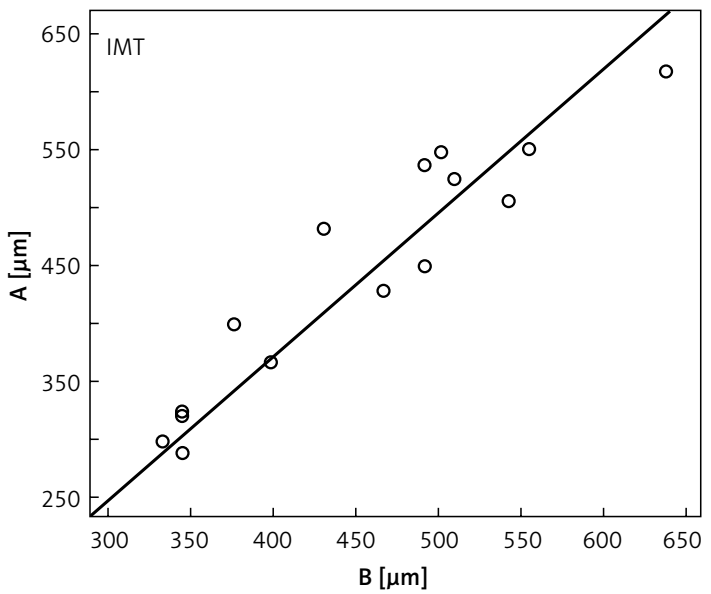

D

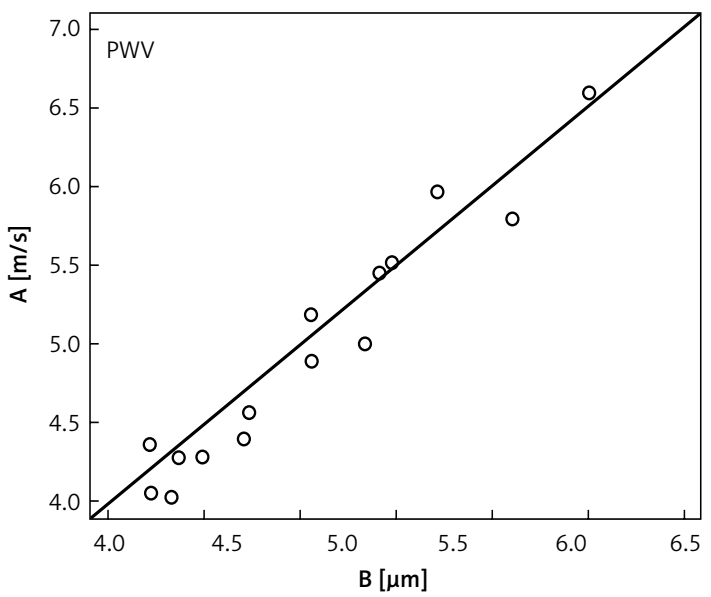

Figure 3. The same physician measured the IMT and PWV at different time points $(\mathrm{A}, \mathrm{C})$, showing the correlation coefficient: $r 1=0.97$ (B), $r 2=0.93$ (D)

thyroid hormones result in over-release of vascular excitatory substances, and cause damage of vascular endothelial cells, ultimately changing the elastic parameters in the blood vessel $[16,17]$. Compliance coefficient is increased after the ${ }^{131}$ treatment and $\beta$ and PWV are decreased compared to before treatment.

Furthermore, our correlational analysis showed that IMT and functional parameters of the carotid artery were exclusively associated with the patients' age, not relevant to hemodynamic factors and thyroid hormones. After controlling the age factor, the partial correlation analysis showed that these parameters were associated with diastolic blood pressure, pulse pressure and heart rate, and not associated with levels of thyroid hormone, suggesting that hemodynamic factors such as blood pressure and heart rate were influential factors for carotid artery function independent of age.

In this study, after the ${ }^{131}$ I treatment, patients had a lower IMT, increased CC and decreased $\beta$ and PWV compared to before treatment, which indicated that both structure and function of the carotid artery recovered in patients with hyperthyroidism, with increased artery compliance, reduced stiffness, and elastic recovery to a normal level. The mechanism of ${ }^{131}$ treatment implicated might be related to the radioactive function on the thyroid itself, which can destroy part of the thyroid and reverse the normal endocrine effect of thyroid. However, the exact mechanism needs further investigation. Other reports showed that IMT of the common carotid artery in patients with ischemic stroke is related to the immune status assessed by the quantity of CD4+ cells in the body, and hyperthyroidism is also an auto-immune disease [18].

In the current study, we found no difference in bilateral carotid arteries regardless of the structural or functional parameters, which indicated that the carotid artery on either side can be investigated in future studies. In addition, we also found that serum thyroid hormone levels were not correlated with the structural and functional damage to the common carotid artery, and we speculated that thyroid hormones have no direct impact on the vascular structure and function, but rath- 
er have an indirect impact on the hemodynamic change. Inaba et al. [14] reported that the gradually reduced $\beta$ in hyperthyroid during treatment was related to the pulse pressure and not associated with thyroid hormone levels, which was consistent with our study.

Based on the clear visualization of the two-dimensional vascular structure, RF-data technique can receive complete RF signal, and detect the carotid IMT in six cardiac cycles in real-time. This technique has many advantages, including non-invasive and high precision, which is up to $10 \mu \mathrm{m}$. This technology is considered as a reliable and feasible method of clinical evaluation of arterial structure and function [19]. In this study, we only recruited hyperthyroid patients without previous treatment for thyroid disorder, and the results showed that the structure and function of the carotid artery in patients before treatment were significantly different compared to healthy controls. After 6 months of treatment with iodine, abnormal parameters recovered to the normal state, which further indicated that the RF data technology can accurately and sensitively evaluate the structure and function of the carotid artery in patients with hyperthyroidism. In addition, the results of the reproducible test further confirmed that the RF data technology could automatically and repeatably monitor the structure and function of the carotid artery in patients with hyperthyroidism, and is conducive to clinically comprehensive assessment of its vascular lesions.

There were also some limitations in this study: the relatively small sample size may, to some extent, reduce the statistical accuracy; and further work has not been carried out for more than 6-months of follow-up after treatment of patients with hyperthyroidism.

In conclusion, 6-month ${ }^{131} \mathrm{I}$ treatment for patients with hyperthyroidism reverses the structural and functional damage in the carotid artery, which is sensitively evaluated by the RF data technique.

\section{Conflict of interest}

The author declares no conflict of interest.

References

1. Klein I, Danzi S. Thyroid disease and the heart. Circulation 2007; 116: 1725-35.

2. Penelope JD, Owen RS. Thyroid disease and vascular function. Thyroid 2007; 17: 519-24.

3. Wang JH, Wang YH, Wu CL. Quantitative evaluation of carotid elasticity in normal adults by echo-tracking technique. Chin J Ultrasonog 2005; 14: 292-4.

4. Lidia O, Malgorzata KK, Andrzej L. Effects of drugs on the efficacy of radioiodine (131I) therapy in hyperthyroid patients. Arch Med Sci 2010; 6: 4-10.

5. Klatika M, Grywalska E, Surdacka A, et al. Peripheral blood lymphocyte apoptosis and its relationship with thyroid function tests in adolescents with hyperthyroidism due to Graves' disease. Arch Med Sci 2012; 8: 865-73.

6. Tu ST, Wang IW, Lin HF. Carotid intima-media thickness and stiffness are independent risk factors for atherosclerotic diseases. J Investig Med 2010; 58: 786-90.

7. Laurent S, Boutouyrie P. Arterial stiffness: a new surrogate end point for cardiovascular disease? J Nephrol 2007; 20: S45-50.

8. Touboul MG, Hennericim M. Carotid intima-media thickness consensus. Cerebrovasc Dis 2007; 23: 75-80.

9. Bahn RS, Burch HB, Cooper DS, et al. Hyperthyroidism and other causes of thyrotoxicosis: management guidelines of the American Thyroid Association and American Association of Clinical Endocrinoligists. Thyroid 2011; 21: 1-54.

10. Faber J, Wiinberg N, Schifter S, et al. Haemodynamic changes following treatment of subclinical and overt hyperthyroidism. Eur J Endocrinol 2001; 145: 391-6.

11. Volzke H, Robinson DM, Schminke UL. Thyroid function and carotid wall thickness. J Clin Endocrinol Metabol 2004; 89: 2145-9.

12. Bilir C, Gökosmanoglu F, Caliskan M, et al. Regression of the carotid intima media thickness by propylthiouracil therapy in Graves' hyperthyroidism. Am J Med Sci 2011; 5: 1-4.

13. Lorenz MW, Polak JF, Kavousi M, et al. Carotid intimamedia thickness progression to predict cardiovascular events in the general population (the PROG-IMT collaborative project): a meta-analysis of individual participant data. Lancet 2012; 379: 2053-62.

14. Inaba M, Henmi Y, Kumeda Y. Increased stiffness in common carotid artery in hyperthyroid Graves' disease patients. Biomed Pharmacother 2002; 56: 241-6.

15. Hamano K, Inoue M. Increased risk for atherosclerosis estimated by pulse wave velocity in hypothyroidism and its reversal with appropriate thyroxine treatment. Endocr J 2005; 52: 95-101.

16. Quesada A, Sainz J, Wangensteen R. Nitric oxide synthase activity in hyperthyroid and hypothyroid rats. Eur J Endocrinol 2002; 147: 117-22.

17. Napoli R, Biondi B, Guardasole V. Impact of hyperthyroidism and its correction on vascular reactivity in humans. Circulation 2001; 104: 3076-80.

18. Drechsler $H$, Masztalewicz $M$, Safranow $K$, et al. CD4+ CD28- lymphocytes and ischemic stroke. Part I: CD4+CD28-lymphocytes and common carotid artery intima-media thickness. Neurol Neurochir Pol 2013; 47: 201-7.

19. Magda SL, Ciobanu AO. Comparative reproducibility of the noninvasive ultrasound methods for the assessment of vascular function. Heart Vessels 2012; 13: 225-32. 